\title{
La utilización de las tecnologías de la información y la comunicación (TIC) en la consolidación de las trayectorias afectivo-sexuales de los jóvenes homosexuales y bisexuales colombianos residentes en España
}

\author{
Jair Eduardo Restrepo Pineda ${ }^{1}$
}

Recibido: 08/08/2016 / Revisado: 15/10/2016/ Aceptado: 24/02/2018

Resumen. La utilización de las redes sociales como herramientas para consolidar las trayectorias afectivo-sexuales de los jóvenes homosexuales y bisexuales colombianos en España se evidencia desde el inicio del proceso migratorio hasta su integración en la sociedad de acogida. Las tecnologías de la información y la comunicación, especialmente internet y las aplicaciones para dispositivos móviles, permiten a los jóvenes migrantes crearse un imaginario y conocer, antes aún de iniciar el proceso migratorio, las pautas y los comportamientos en materia afectivo-sexual del país de destino, lo cual facilita su integración. Sin embargo, esto no sucede en todos los casos ya que la información que se intercambia no necesariamente resulta veraz u objetiva. La utilización de las TIC por este segmento de población es generalizada, además de que es el medio más eficiente y eficaz para la transmisión de información útil para mejorar la calidad de vida de estos jóvenes.

Palabras clave: TIC; migración; sexualidad; joven; homosexuales.

\section{[en] The use of ICTs in consolidating affective-sexual life pathways of young Spanish- resident homosexual and bisexual Colombians}

\begin{abstract}
The use of social networks as tools for consolidating the affective-sexual life pathways of young homosexual and bisexual Colombians in Spain is clear from the outset of the migratory process until their integration into the host society. Even before young migrants embark on the migratory process, ICTs and particularly the Internet and mobile phone apps allow them to develop an image of and to understand the affective-sexual norms and conduct of the host country, aiding their integration. This does not happen in all cases, however, as the information exchanged is not necessarily accurate or objective. The use of ICTs is widespread among this segment of the population, in addition to being the most efficient and effective means for the transmission of useful information that allows these young people to improve their quality of life.
\end{abstract}

Keywords: ICT; migration; sexuality; youth; homosexual.

Sumario: Introducción. 1. Metodología. 2. Resultados. 2.1. Las trayectorias sexuales. 2.2. El encuentro con Chueca y la vida gay en España. 2.3. Nuevos contextos sociales, culturales y sexuales en España. 2.4. Los espacios virtuales. 2.5 Medios de información sobre salud sexual y reproductiva. 3. Conclusiones. 4. Referencias bibliográficas.

Cómo citar: Restrepo Pineda, J. E. (2018) La utilización de las tecnologías de la información y la comunicación (TIC) en la consolidación de las trayectorias afectivo-sexuales de los jóvenes homosexuales y bisexuales colombianos residentes en España, en Cuad. trab. soc. 31(2), 529-542.

\footnotetext{
Corporación Universitaria Minuto de Dios UNIMINUTO Sede Bello, Colombia jair.restrepo@uniminuto.edu
} 


\section{Introducción}

La utilización de dispositivos electrónicos, como los teléfonos móviles, las tabletas y los computadores, y todas las aplicaciones vinculadas a estos, han permitido crear un nuevo espacio de socialización para los varones jóvenes homosexuales y bisexuales colombianos residentes en España. Estos dispositivos y sus aplicaciones se usan para entablar amistad, buscar sexo ocasional o pareja estable.

El uso de estas herramientas se halla ampliamente extendido entre los inmigrantes entrevistados y todos utilizan por lo menos uno de estos espacios para socializar con otros hombres. Además, internet es una de las herramientas más utilizadas por estos varones para acceder a información sobre la comunidad LGBTI en España, en especial, a locales de diversión y ocio, fiestas y noticias en general, así como para consultas sobre el virus de inmunodeficiencia humana (VIH/ SIDA), y las infecciones de transmisión sexual (ETS).

Es así como las tecnologías de la información y la comunicación (TIC) son mucho más que un conjunto de técnicas, desarrollos $\mathrm{y}$ dispositivos que integran funcionalidades de almacenamiento, procesamiento y transmisión de datos; ya que cumplen una función mediadora entre los individuos que les permite entablar relaciones afectivas o sexuales, pero que además facilita la vida de los involucrados en este proceso. Es así como:

Los jóvenes inmigrantes necesitan construir una identidad propia en la que sus raíces puedan coexistir con los nuevos valores resultantes de la aculturación. Para que estos jóvenes puedan alcanzar el éxito en su integración, la cultura anfitriona debe necesariamente crear Espacios de Encuentro en los que el intercambio de información y las actividades comunes de ocio y tiempo libre potencien la creación y el desarrollo de culturas híbridas (Hernández y Alcoceba, 2015, p. 122).

Por lo tanto, las tecnologías de la información y la comunicación entran a jugar un papel decisivo en la sexualidad, entendida como «el medio por el cual la gente intenta ser consciente de sí misma, es decir, de constituir su identidad como sujetos» (Sennet y Foucault, 1981, p. 47). En los casos en que los individuos se encuentran inmersos en otros contextos sociales y culturales, las TIC adquieren una mayor relevancia a la hora de construir o redefinir esa identidad sexual.

El artículo tiene como objetivo evidenciar el papel que cumplen estas tecnologías dentro de las trayectorias afectivo-sexuales de los jóvenes colombianos residentes en España, y como dichas herramientas mejoran la integración de los migrantes en la sociedad de acogida; pero además, pretende poner de manifiesto el potencial que tienen estos medios para desarrollar programas de promoción y prevención en la salud sexual y reproductiva de los jóvenes.

Según la Organización Mundial de la Salud, la sexualidad es un aspecto central del ser humano, presente a lo largo de su vida. Abarca al sexo, las identidades y los papeles de género, el erotismo, el placer, la intimidad, la reproducción y la orientación sexual. Se vive y se expresa a través de pensamientos, fantasías, deseos, creencias, actitudes, valores, conductas, prácticas, papeles y relaciones interpersonales.

La sexualidad es un medio generalizado de comunicación entre las personas cuyas expresiones varían dependiendo del contexto social donde se encuentren, pero que persisten como un recurso indispensable en todos los contextos sociales.

El estudio de las relaciones personales y, entre ellas, las afectivo-sexuales son elementos indispensables para conocer quiénes somos, porque la sexualidad se convierte en el medio a través del cual las personas intentan ser conscientes de sí mismas y de construir su identidad como sujetos y les permite, a su vez, establecer relaciones sociales con los otros individuos.

Es así como se da inicio a - lo que algunos autores han denominado - la tecnosociabilidad; es decir:

[La] experiencia cotidiana y recurrente de intercambio e intersubjetividad asociada al uso de los medios horizontales de comunicación de masas (Internet, celulares y sus múltiples utilizaciones y constantes actualizaciones) está modificando los patrones de conocimiento y de aprendizaje, y ello se expresa en las diversas dimensiones de la cotidianidad: trabajo, estudio, hogar, sexo, divertimento. Es decir, se trata del uso de estas tecnologías no como herramientas o fines, sino como ámbitos que permiten nuevas formas de ser, espacios donde se ponen en juego valores, se construyen identidades y se expre- 
san sensibilidades culturales, sociales, ecológicas y estéticas" (Calderón, 2013, p.1).

Como lo pone de manifiesto claramente Calderón, las TIC se convierten en espacios donde se construyen y redefinen las identidades de los individuos, gracias al intercambio permanente de conceptos, percepciones, ideas y valores, donde la sexualidad no se encuentra ajena a este proceso. Además, son los jóvenes quienes utilizan de forma constante estos nuevos espacios de sociabilidad. Según la legislación colombiana, se considera joven a: "toda persona entre 14 y 28 años cumplidos en proceso de consolidación de su autonomía intelectual, física, moral, económica, social y cultural que hace parte de una comunidad política y en ese sentido ejerce su ciudadanía" (Dirección del Sistema Nacional de Juventud, 2013, p.5).

En el caso de los jóvenes entrevistados en España, el encuentro con nuevos contextos sociales, culturales y sexuales se refieren no solo a las trayectorias sexuales, sino a las nuevas formas de relacionarse y establecer otro tipo de vínculos afectivos diferentes a aquellos que los migrantes conocían en su país de origen. Es en este proceso donde las TIC cobran su mayor importancia ya que les permiten a los varones acceder a información rápida y precisa para conocer y experimentar otros espacios de homosocialización. Tanto la creación de nuevos vínculos personales como el conocimiento de otros espacios sociales se convierten en las primeras etapas del proceso de integración del migrante en la sociedad de acogida.

Esta nueva gama de formas de ver y de vivir la sexualidad que esta mediada en muchos casos por las TIC pone al migrante en un proceso constante de cambio y adaptación en lo que García Canclini (1989) ha denominado el «proceso híbrido», pues los migrantes advierten que en la sociedad española las relaciones afectivo-sexuales tienen otros mecanismos y otros significados diferentes a los de su país de origen, tal como se ha podido constatar en las entrevistas realizadas.

Finalmente, las TIC deben ser aprovechadas por su potencial como herramientas para la promoción y la prevención en salud sexual y reproductiva, especialmente para los jóvenes homosexuales y bisexuales. Puesto que su utilización en este segmento de la población resulta ser permanente, además de ser más eficiente y eficaz para la transmisión de informa- ción útil para mejorar la calidad de vida de los jóvenes.

\section{Metodología}

El artículo se basa en un estudio que incorpora elementos tanto cualitativos como cuantitativos, en el cual se empleó la metodología denominada «bola de nieve», limitando los referidos de cada uno de los entrevistados a tres personas, lo que permitió incluir individuos de diferentes edades, condiciones sociales, niveles educativos y profesionales, logrando así diversificar y profundizar en redes sociales más heterogéneas. El muestreo por «bola de nieve» consintió paliar la utilización de «sistemas de muestreo que incluyen sesgos más sutiles, como utilizar muestras de sujetos que se ofrecen voluntarios, o solo de aquellos miembros de colectivos activistas o asistentes a actos filo-gais o bares específicos» (Baile, 2008, p.24). En estos casos, los estudios sobre homosexualidad abusan de muestras de fácil acceso que, según Baile, generan dudas sobre la posible generalización de los resultados, ya que se concentran en segmentos de la comunidad gay que comparten características muy similares, dejando de lado aquellos que no comparten estas.

Antes de iniciar el muestreo por «bola de nieve» se definieron espacios estratégicos, de los que se seleccionaron aleatoriamente los primeros sujetos; la definición de estos sitios pretendía diversificar las características sociales y culturales de los individuos por entrevistar, y profundizar de esta manera en sus redes sociales. Estos sitios fueron: discotecas, saunas, organizaciones no gubernamentales de LGBTI, universidades y zonas de cruising. En estos espacios se seleccionaron aleatoriamente los entrevistados, y a partir de estos se iniciaron las cadenas de referencia.

Para la recolección de la información se utilizó la entrevista en profundidad, en la cual se indagaba sobre aspectos referidos a los medios y herramientas utilizadas por los jóvenes para establecer relaciones de amistad, afectivas o sexuales con otros varones. Además, se incluyeron preguntas referidas a los medios utilizados para acceder a información sobre salud sexual y reproductiva, cultura, diversión y entretenimiento.

La información obtenida en las entrevistas fue sistematizada y analizada con la ayuda del software Atlas.ti, el cual permitió eliminar la 
información irrelevante, identificar las categorías emergentes y relacionarlas con las categorías establecidas al inicio de la investigación. Además, esta herramienta es útil para aproximarse al análisis de los datos identificando los significados potenciales de los mismos.
En el Diagrama 1 se pueden observar las relaciones y las categorías con las que se realizaron los análisis de las entrevistas que fueron respondidas por los jóvenes migrantes colombianos residentes en España. 


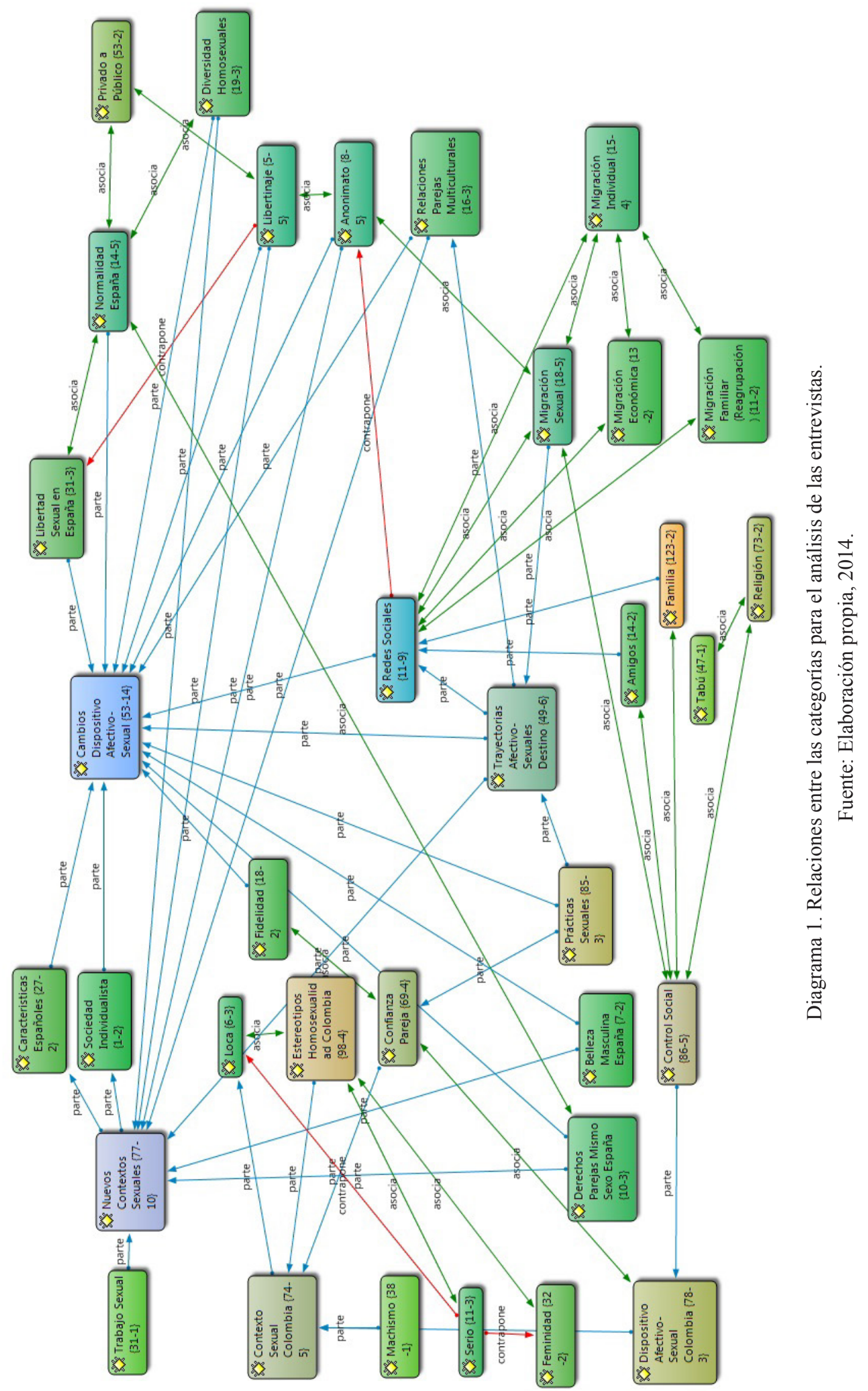




\section{Población objetivo}

La población objetivo es de jóvenes varones mayores de 18 años que se autoidentifican como homosexuales o bisexuales y cuyo lugar de origen es Colombia; con una experiencia migratoria en España de dos años por lo menos. La muestra seleccionada es intencionada y no probabilística, por lo tanto no responde a una estricta cuestión de diseño y planificación, sino al hecho de considerar la saturación de la información recopilada a través de las entrevistas, buscándose fundamentalmente la profundidad, en la que es privilegiada la calidad de la muestra sobre la cantidad. De esta manera, cuando se estimó que se habían cumplido las exigencias de heterogeneidad y variaciones suficientes en la muestra se dio por terminada la realización de las entrevistas.

En total se entrevistó a 26 jóvenes, con una edad promedio de 24 años. El 96 por ciento se encontraban solteros y solo el 4 por ciento estaban casados. En cuanto al nivel académico, el 50 por ciento de los entrevistados han concluido el bachillerato, el 26 por ciento cursaron estudios universitarios, el 12 por ciento la educación media, el 8 por ciento tiene un posgrado, y solo el 4 por ciento han cursado un grado técnico. El 88 por ciento de los jóvenes entrevistados se auto-identifican como homosexuales, frente al 12 por ciento que lo hacen como bisexuales. El promedio de residencia en España es de 4 años y los entrevistados habitan en las comunidades autónomas de Madrid, Valencia, Cataluña y Andalucía.

\section{Resultados}

\subsection{Las trayectorias sexuales}

Las trayectorias sexuales, tanto en Colombia como en España, son muy variadas, y se pueden definir como las rutas, las formas y los sitios de encuentro tanto públicos como privados en los cuales los jóvenes homosexuales y bisexuales establecen relaciones sexuales o afectivas. La iniciación sexual se da generalmente en el ámbito familiar o con amigos de forma consensuada y de exploraciones mutuas, durante las cuales, por lo menos los varones entrevistados, tienen definida su identidad como homosexuales o bisexuales; sin embargo, se carece de expectativas de roles sexuales específicos.
En los últimos años, la inclusión de las tecnologías de la información y la comunicación en los procesos sociales, fundamentalmente en aquellos encaminados a conocer y establecer relaciones personales, han adquirido un valor preponderante, especialmente en los casos de los varones homosexuales y bisexuales, los cuales encuentran en estas herramientas un medio que les ofrecen seguridad y anonimato.

Los entrevistados en España han hecho mención a las diversas formas de entablar contacto con otros varones que van desde el círculo de amigos hasta los sitios de homosocialización como saunas, bares o discotecas. Un entrevistado manifiesta:

A la primera pareja que fue con la que más duré la conocí por medio de un amigo que él le conoció por internet, me lo ha dicho, la segunda le conocí por internet y la tercera también por medio de una amiga (Emilio, 26 años, Madrid).

\section{Otro interlocutor sostiene:}

Yo los conocí por un chat y luego pues evidentemente, lo que generalmente se hace se conocen las personas por internet, luego se conocen en persona y a lo mejor hay una química entre esas dos personas y bueno, ya lo demás lo que surja (Vicente, 27años, Valencia).

La utilización de internet está generalizada, ya sea a través de los chat o perfiles en páginas para homosexuales o bisexuales o bien como aplicaciones para los dispositivos móviles como Bender (http://www.benderapp.com/) y Grindr (http://grindr.com/), que permiten contactar con otros hombres de manera ágil y eficaz. En palabras de un joven:

Aquí son más tirantes, aquí te pillo y aquí te mato y nunca más te volví a hablar, allá en Colombia son más afectivos, bueno en mi caso, no lo sé en otra persona (Ángel, 28 años, Valencia).

Estos medios son considerados por los jóvenes migrantes como menos personales y más funcionales, lo cual choca con una percepción de las relaciones más íntimas construida en el país de origen; además, algunos de ellos piensan que las relaciones personales y sociales en España son más individualistas en comparación con aquellas que se establecen en Colombia.

Sin embargo, 
Los cambios comunicativos asociados a dichas innovaciones están creando nuevos espacios para la relación, más allá de la dimensión presencial real. Así, la aparición de las redes virtuales ha venido a reconfigurar los ámbitos de actuación y de relación juveniles, tanto en sus dimensiones productivas (tareas escolares, formación, etc.), como reproductivas (ocio y relaciones familiares y personales) (Hernández y Alcoceba, 2015, p. 128).

Ahora bien, los jóvenes inmigrantes se encuentran con nuevos espacios de homosocialización y, por lo tanto, nuevos trayectos que no conocían en su país de origen, pues en España se ha dado un proceso de apropiación de espacios que han llevado:

Incluso a volver privados los actos públicos (por ejemplo el baile en las fiestas exclusivas), y públicos los que la sociedad considera privados (encuentros sexuales en sitios como cines), con lo que se hace difícil aprehender la multiplicidad de funciones y significados de la vida gay urbana (List, 2005, p. 196).

Todo ello configura un nuevo contexto sexual al que los varones inmigrantes se deben enfrentar, brindándoles una variedad de experiencias sexuales y una diversidad de espacios para llevar a cabo estos. Se pone en marcha un proceso de reedición de las percepciones y los significados de la sexualidad, propios de su país de origen; así, "cuando se da una combinación o mezcla de contextos culturales diferentes se produce un proceso de hibridación que genera nuevas estructuras, objetos y prácticas" (Hidalgo y Flores, 2008, p. 33).

La reedición de los significados sobre la sexualidad debe entenderse como proceso cultural dinámico y no acabado, en el cual "los individuos pueden entrar o salir según el contexto cultural en el que se encuentren y que les da la posibilidad de integrarse - mas no de asimilarse- a otros contextos culturales" (idem).

\subsection{El encuentro con Chueca y la vida gay en España}

El tipo de contacto que han tenido los inmigrantes en su país de origen con comunidades LGBTI influye en la forma en la que encuentran y establecen vínculos con estas en España. Estos contactos son de diversa índole y pueden incluir: relaciones de amistad, mass media, participación en clubs o actividades sociales, experiencias educativas, actividades de ocio y, de manera generalizada, internet y la utilización de aplicaciones para dispositivos móviles, que no solo les permiten ubicar sitios de encuentro, sino que además facilitan el encuentro con otros jóvenes.

Así, algunos de los entrevistados el único contacto que habían tenido con el colectivo gay en su país de origen era a través de las redes sociales, y los referentes la comunidad LGBTI en España los habían adquirido a través de la televisión e internet.

Los primeros acercamientos que se establecen en el país de destino se realizan a través de amigos, quienes en algunas ocasiones son contactados a través de redes sociales; estos guían a los varones para que conozcan y se aproximen de una manera más acertada al nuevo contexto sexual que les ofrece una ciudad como Madrid, (y específicamente, el barrio de Chueca, zona que es ampliamente reconocida como el principal barrio gay de la capital y un referente para el colectivo LGBTI en España). Un joven narra su experiencia:

Conocí una persona por internet y me trajo, yo cuando vivía en Collado Villalba yo no conocía Madrid. Este chico me invitó a que conociera Chueca y me explicó más o menos donde no tenía que meterme, cuáles eran los sitios buenos y cuáles los malos. Cuáles eran los fuertes y los no fuertes y con él aprendí un poco el movimiento de discotecas y bares (Samuel, 28 años, Madrid).

Se evidencia el papel que cumplen las TIC como medio para establecer vínculos con otros jóvenes homosexuales o bisexuales que finalmente permitirán la incorporación del migrante al circuito LGBTI en España y, por lo tanto, su integración a la sociedad de acogida. Otros jóvenes en cambio, con experiencias más amplias en su país de origen, toman la iniciativa para conocer y contactar con el ambiente gay en su ciudad de residencia, ya sea utilizando aplicaciones para dispositivos móviles o navegando por internet; siendo esta última opción la forma más extendida de búsqueda de aquellos sitios o zonas que les pueden resultar interesantes. Además, debido a la percepción de seguridad que tienen en el país de destino, realizan citas con otros individuos a través de estas aplicaciones o recurriendo a perfiles en páginas web destinadas para tal fin. En palabras de un entrevistado: 
Por internet, busqué lugares de ambiente en Madrid y obtuve la información. Pues bueno, la verdad es que fue una experiencia agradable, porque no te esperas encontrar ese ambiente, ya que no estás acostumbrado a verlo, tienes nuevas experiencias y te agrada, te sientes más seguro de estar en esos lugares porque no hay nadie que te vaya a mirar mal, todos son como tú y claro no hay ninguna discriminación hacia ti porque frecuentes esos sitios (Sergio, 19 años, Madrid).

Además, estos jóvenes tenían referencias previas sobre el contexto sexual y de género en España que habían recibido a través de sus familiares y amigos antes de iniciar el proceso migratorio, lo que les facilitó el acceso a información que utilizaron para iniciar su búsqueda e integración en los circuitos gay de sus ciudades de residencia.

Internet se constituye como una de las fuentes de información sobre el nuevo contexto social y sexual en el país de destino; es así como en todos los casos los jóvenes homosexuales o bisexuales entrevistados han utilizado esta herramienta para conocer aspectos puntuales tales como direcciones de sitios de homosocialización, los precios de los servicios, los medios de transporte para llegar a estos, entre otros. Es así como

Las redes sociales son un espacio de socialización necesario para los jóvenes: se han convertido en lugares de encuentro y de relación, en los que introducirse, construir la propia identidad y auto-representarse, compartir gustos y hobbies, y adquirir conocimientos y habilidades para el desarrollo personal y social (Stern, 2004; Tortajada, Araüna, y Martínez, 2013; Manago et al., 2008, citado en Dueñas et al., 2016, p. 69).

El primer contacto con la comunidad gay en España resultó ser satisfactorio y agradable para estos varones, que se integraron en un ambiente que no resultaba hostil y que les permitió incorporarse rápidamente a este estilo de vida. Para aquellos varones que residen en la Comunidad de Madrid, la zona de Chueca se convierte en el punto de referencia de la vida LGBTI; esta zona se ubica en el barrio de Justicia, situado en el distrito centro de la ciudad.

Las experiencias de los varones homosexuales y bisexuales vividas en su país de origen determinarán la forma en la que estos se integren y participen en el nuevo contexto social y sexual de España. El proceso vincula de manera permanente tanto los países de origen como de destino, o en otras palabras: los contextos sociales de los jóvenes en Colombia influyen en su integración en la comunidad LGBTI en España y los tipos de vida gay a los que tienen acceso.

Es en este proceso de intercambio constante de información entre los países de origen y de destino donde las TIC cumplen su papel fundamental que permite a los varones sin experiencia migratoria crear un imaginario y una idea preconcebida sobre el contexto sexual, social y cultural del lugar de destino, que en mayor o menor medida podrá determinar la forma como se integrarán en todos estos ámbitos.

\subsection{Nuevos contextos sociales, culturales y sexuales en España}

El encuentro con nuevos contextos sociales, culturales y sexuales en España se refieren no solo a las trayectorias sexuales, sino a las nuevas formas de relacionarse y establecer otro tipo de vínculos afectivo-sexuales, diferentes a aquellos que los migrantes conocían en su país de origen, además de conocer y experimentar otros espacios de homosocialización, algunos de los cuales tienen características muy específicas referidas al tipo de personas que los frecuentan y las prácticas sexuales que allí se realizan.

De esta manera,

El cambio de contextos, producto de la migración, origina que el inmigrante se enfrente a una nueva serie de normas y pautas sociales que en la mayoría de las ocasiones resultan ser muy diferentes a las que guiaban sus encuentros sexuales en Colombia. Tales contextos determinan, además, el acceso a relaciones casuales o a la consolidación de las relaciones estables, así como todo el proceso de incorporación dentro de la comunidad LGBTI en destino (Restrepo, 2013, p. 44).

Los jóvenes inmersos en estos nuevos contextos sociales y culturales han podido adaptarse a la nueva cultura sexual sin que ello signifique un proceso de asimilación, como lo corroborarían aspectos tales como las relaciones de pareja basadas en la confianza y el conocimiento previo de la persona, entre otros elementos. 


\subsection{Los espacios virtuales}

Estos espacios se centran en la utilización de las TIC, especialmente internet y las aplicaciones para dispositivos móviles, como medio de socialización por los jóvenes homosexuales y bisexuales colombianos residentes en España cuya finalidad puede ser entablar amistad, buscar sexo ocasional o pareja estable. Un entrevistado afirma:

Internet, única y exclusivamente internet sobre todo el chat, en aquel entonces no había ciertas cosas como las que hay hoy, como el whats app y las aplicaciones para móviles, en aquel entonces solamente en internet, páginas de chat era donde buscaba chicos» (Nelson, 23 años, Valencia).

Entre los espacios virtuales más utilizados por estos varones, tenemos los chats (www. chueca.com), los perfiles en páginas web destinados a homosexuales y bisexuales (www. manhunt.net; www.planetromeo.com; www. bakala.org; www.tuamo.net) y las aplicaciones para dispositivos móviles anteriormente indicadas. El uso de estas herramientas se halla ampliamente extendido entre los inmigrantes entrevistados ya que todos utilizan, por lo menos, uno de estos espacios para so- cializar con otros hombres. Otro joven manifiesta:

Poco a poco fui conociendo vía internet, ya que en esa época no conocía gente, conocía poquito pero no tenía ni idea de cómo se movía la situación aquí» (Samuel, 28 años, Madrid).

Además, internet es una de las herramientas más utilizadas por estos jóvenes para acceder a información sobre la comunidad LGBTI en España, en especial la referente a locales de diversión y ocio, fiestas y noticias en general, así como consultas sobre VIH/Sida e infecciones de transmisión sexual.

Los datos obtenidos en las entrevistas muestran un uso extendido y constante de internet por los jóvenes colombianos residentes en España, pues el 73 por ciento de estos visitaron alguna página para homosexuales o bisexuales en los dos días anteriores a la realización de la entrevista. Esta información corresponde con los datos recopilados en la investigación Internet y riesgo sexual en hombres que tienen sexo con hombres, realizada en España, según la cual "un $14 \%$ de los hombres que tienen sexo con hombres encuestados en lugares de ambiente refirió no usar nunca Internet" (Fernández y Zaragoza, 2009, p. 382), es decir que el 86 por ciento de los mismos sí hacen uso de esta herramienta.

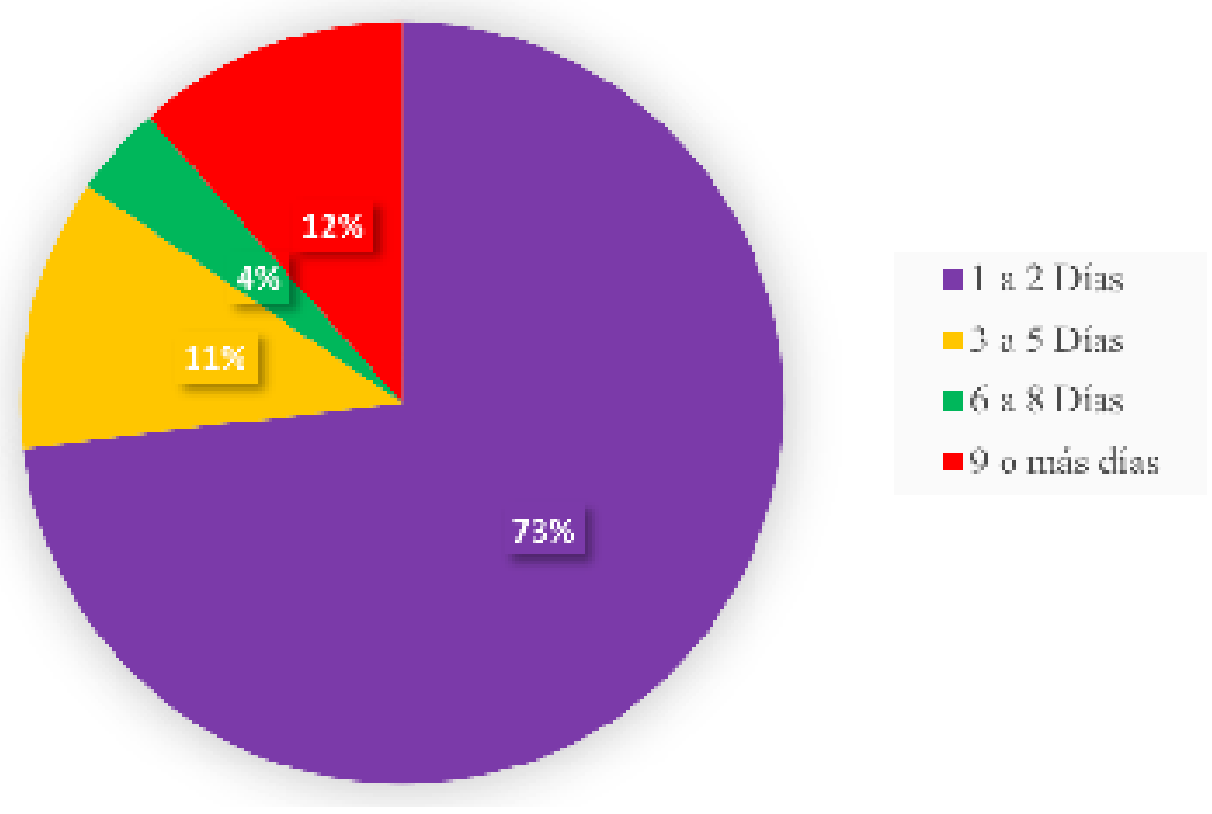

Gráfico 1. Porcentaje de jóvenes encuestados según la fecha de la última visita a alguna página web con contenidos específicos destinados a esta población.

Fuente: Elaboración propia, 2014. 
Por otra parte, el 88 por ciento de los jóvenes visitan por lo menos una vez a la semana algún tipo de página web con contenidos para homosexuales o bisexuales, siendo este un recurso potencial para desarrollar campañas de promoción y prevención en salud para este colectivo.

Esta situación pone de manifiesto un cambio en los patrones de socialización entre los jóvenes homosexuales y bisexuales, en la cual se pasa de encuentros más íntimos y personales a aquellos mediados por los dispositivos móviles, escenario que ya se había evidenciado en otros estudios según los cuales la "Internet puede suplir el rol de los bares, las saunas, las discotecas, etc., al proporcionar a los hombres que tienen sexo con hombres oportunidades de relacionarse on-line y de realizar una amplia variedad de conductas sexuales" (Fernández y Zaragoza, 2009, p.381). En palabras de un joven:

Ahora mismo por internet que se hace todo mucho más fácil. Hay un chico que me gusta y paso mucho por ahí, alguna vez he hablado con él, pero tampoco hemos profundizado nada y no ha pasado nada» (Octavio, 22 años, Valencia)

Los inmigrantes entrevistados consideran que internet facilita los encuentros con otros jóvenes, lo que permite un conocimiento previo de la persona $y$, por lo tanto, se encuentra mejor valorado como medio de socialización que otro tipo de espacios como los bares, las discotecas o los locales de sexo.

\subsection{Medios de información sobre salud sexual y reproductiva}

Se puede considerar que el nivel de conocimiento e información de todos los jóvenes entrevistados con respecto a las infecciones de transmisión sexual, incluyendo el VIH/Sida, y las prácticas para prevenirlas es alto. Precisamente, "la educación para el sexo seguro, que permitió pensar en el sexo y la sexualidad en función de las prácticas y no de las identidades. En una palabra, lo importante era lo que se hacía, no lo que uno era" (Spargo, 2007, p.46).

Sin embargo, aunque los entrevistados hablan claramente del sexo seguro, algunos han incurrido prácticas de riesgo que se encuentran vinculadas específicamente con situaciones y contextos nuevos para ellos y en los que, además, han intermediado otros factores como: las sustancias psicoactivas o el desconocimiento de las normas que rigen tales encuentros.

Siguiendo a Restrepo (2016), podemos decir que no solamente las trayectorias sexuales o el descubrimiento de espacios de homosocialización, que están mediados en la mayoría de los casos por la utilización de aplicaciones para dispositivos móviles, pueden ser nuevos para los jóvenes inmigrantes; también lo son las relaciones afectivo-sexuales que establezcan con otros varones, ya sean de su propia o de diferente nacionalidad, pues el contexto social, cultural y sexual del país de destino condicionará de manera específica dichas relaciones.

En los discursos de los entrevistados se observa que la utilización del preservativo está condicionada por la confianza que brinda el conocimiento previo de la otra persona, así como por la percepción sobre su comportamiento. Sin embargo, se hacen explícitas las dudas y aun así no se utiliza el preservativo, ya que "en las relaciones de pareja estable el uso del preservativo connota falta de confianza de ambas partes" (Hidalgo y Flores, 2008, p.43). Los siguientes testimonios así lo evidencian:

Hombre, no utilizaba el condón, si era por ejemplo con mi pareja, pues en eso había menos duda y decidíamos sin condón, pero si es por ejemplo, con el chico de la disco que conozco esa noche con condón (David, 20 años, Madrid).

Al principio lo utilizábamos a medida que no sé... de que estábamos más compenetrados lo dejamos de utilizar. Luego de todas formas me hice la prueba, por eso la primera vez que me la hice fue por eso, como estaba manteniendo relaciones continuamente con él, pues me la hice por si acaso (Octavio, 22 años, Valencia).

Los dispositivos móviles y sus aplicaciones cumplen dos funciones fundamentales para los jóvenes entrevistados: la primera, es su utilización como medio para acceder a información sobre lugares de encuentro que les permiten establecer contactos personales con otros varones. En segundo lugar, como fuente de información sobre salud sexual y reproductiva. Es así como los jóvenes migrantes entrevistados en España prefieren acceder a esta información a través de internet, el 46 por ciento, después se encuentran las consultas al médico, el 27 por ciento, y le siguen las asociaciones y organizaciones no gu- 
bernamentales, el 19 por ciento; finalmente se encuentran los amigos, el 8 por ciento. Además, según otros estudios realizados en España, "en cuanto a la promoción de la prueba del VIH, hubo una buena percepción a ser contactado por teléfono o en un local, ya que generaba sensación de ser cuidado" (Jacques, García, Díez, Martín y Cayla, 2015, p. 255).
En este mismo sentido, las TIC deberían convertirse en instrumentos que permitieran a los profesionales "examinar las formas en las que los inmigrantes pueden participar más activamente en redefinir los contextos sexuales en los que interactúan, y no solamente ser sujetos de las normas que condicionan dichos contextos" (Restrepo, 2016, p. 24).

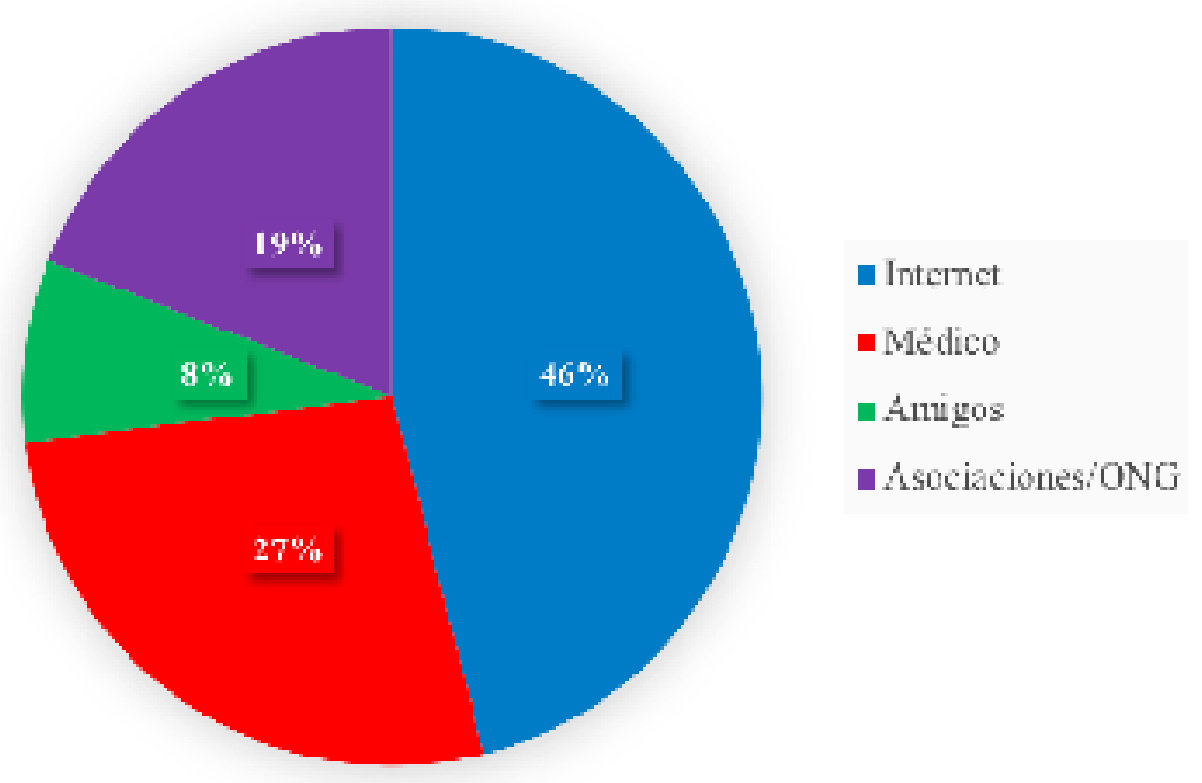

Gráfico 2. Porcentaje de jóvenes entrevistados según la fuente de información consultada en temas de salud sexual y reproductiva.

Fuente: Elaboración propia, 2014

Estos datos ponen de manifiesto la importancia que han adquirido las TIC frente a la promoción y prevención en salud sexual y reproductiva y la necesidad de que la información que se encuentra disponible a través de estos medios sea clara, precisa y confiable.

La utilización de las nuevas tecnologías de la información y la comunicación se ha convertido, pues, en una herramienta fundamental para crear y consolidar redes sociales, y en uno de los principales medios a través de los cuales los jóvenes homosexuales y bisexuales logran establecer relaciones sexuales y sexo-afectivas. La mayor parte de los entrevistados han hecho mención de internet para tal fin, y para ello cuentan por lo menos con un perfil en alguna página de contactos personales o acceden a algún chat.

Las páginas de contactos personales, los chats, el correo electrónico, los grupos de discusión y los foros, así como las páginas web destinadas específicamente a la comunidad
LGBTI se convierten en una fuente de información importante porque permiten a sus usuarios mantener un anonimato, brindándoles a su vez la oportunidad de expresar sus conceptos, ideas, intereses y expectativas frente a su realidad social, afectiva y sexual.

Todos estos aspectos ponen de manifiesto el papel que cumplen las TIC en el quehacer del trabajador social, ya que estas tecnologías permiten la sistematización, el análisis y la difusión de los datos de manera más eficaz y eficiente mejorando la labor de estos. De esta manera, las TIC constituyen un espacio para realizar la intervención social, ya que, por ejemplo, en el caso de los varones homosexuales y bisexuales sería posible establecer relaciones más horizontales entre estos y el profesional, posibilitando una mayor confidencialidad $y$, por ende, un intercambio de información veraz y objetiva.

En este proceso, las TIC no solo deben facilitar el encuentro entre varones con diversas 
orientaciones sexuales, sino que además deben permitir a los profesionales de las ciencias sociales y humanas acercarse a sus realidades, estudiarlas y brindar soluciones a las problemáticas que se identifiquen.

En el caso estudiado de los jóvenes colombianos migrantes en España, resulta evidente que un uso adecuado de las TIC puede ampliar considerablemente su capital relacional y, por ende, los beneficios en su integración social, de manera que puedan evitarse situaciones de exclusión. Sin embargo, un uso inadecuado de estas tecnologías puede conducirlos a asumir riesgos y situaciones para las cuales no necesariamente podrían estar preparados. Son precisamente estos escenarios los que se deben considerar en la intervención social, de tal manera que se ofrezca información precisa y ajustada a los contextos sociales en los cuales estos jóvenes establecen sus relaciones afectivo-sexuales en el país de destino.

Por otra parte, la utilización generalizada por los varones homosexuales y bisexuales de este tipo de tecnologías es una ventaja que se debe considerar a la hora de diseñar e implementar proyectos de intervención social, ya que las TIC son más flexibles y accesibles y brindan una mayor fluidez en la comunicación entre los interlocutores. Siendo estos aspectos ampliamente valorados por los jóvenes, como se comprueba al cuestionarlos sobre los medios de información que utilizan para acceder a los temas de salud sexual y reproductiva, donde cerca del 50 por ciento de los entrevistados manifiestan que su primera opción es internet.

\section{Conclusiones}

Las tecnologías de la información y la comunicación permiten de diversas formas consolidar las trayectorias afectivo-sexuales de los jóvenes homosexuales y bisexuales colombianos que han migrado a España, su utilización no se limita al momento de incorporarse a la sociedad de acogida, sino que se da aun antes de iniciar el proceso migratorio. Así, internet es una de las herramientas más utilizadas para conocer las realidades del país de destino, especialmente en los ámbitos sociales y culturales, mientras que las aplicaciones para dispositivos móviles les permiten establecer contactos afectivos y sexuales con otros varones en el país de destino. Ambas funciones facilitan la integración de estos jóvenes en España, pero no se limitan a estas cuestiones, sino que van más allá sirviendo de medios para acceder a información sobre salud sexual o reproductiva.

Sin embargo, la información que se intercambia a través de estos medios no necesariamente resulta veraz u objetiva. Ello se debe a que la información sobre los temas sexuales y afectivos suele estar influida por percepciones e intereses personales, lo que podría distorsionar la realidad con la cual el migrante se encontrará en el país de destino, dicha distorsión puede generar respuestas equivocas a situaciones que pondrán al migrante en situación de vulnerabilidad.

Para los jóvenes inmigrantes internet se convierte en la principal fuente de consulta para responder a preguntas referentes a su salud sexual y reproductiva y, por lo tanto, es fundamental crear espacios de consulta que cumplan con las normas de calidad en sus contenidos, que lleven a los internautas a resolver sus inquietudes y que no generen otras nuevas, brindando información poco precisa o distorsiona.

Los inmigrantes entrevistados consideran que internet y las aplicaciones para dispositivos móviles facilitan los encuentros con otros varones, lo que permite un conocimiento previo de la persona $\mathrm{y}$, por lo tanto, se encuentra mejor valorado como medio de socialización que otros espacios como: los bares, las discotecas o los locales de sexo. Es así, como días tras día internet, los dispositivos móviles, los chats y las páginas de perfiles personales para establecer relaciones afectivo-sexuales ganan terreno con relación a las trayectorias tradicionales (bares, discotecas u otros sitios de homosocialización) ya que resultan más económicos, más agiles y más eficientes.

Es importante destacar, además, que los jóvenes entrevistados recurren a internet en búsqueda de información sobre salud sexual y reproductiva, por lo tanto debe reconocerse esta herramienta como un medio prioritario para emprender campañas de promoción y prevención de las infecciones de transmisión sexual y del VIH/Sida, ya que es la primera fuente de información a la que recurren estos varones.

Además, se hace necesario que la información que esté disponible a través de este medio sea veraz, precisa y actualizada, que brinde a los jóvenes orientación efectiva para determinadas situaciones. De tal manera que estos espacios virtuales y las aplicaciones para dispositivos móviles a las cuales acceden de manera casi diaria los entrevistados se puedan 
aprovechar para desarrollar intervención social e investigación.

Finalmente, se constata como las TIC, y específicamente las aplicaciones para dispositivos móviles y páginas web destinadas a varones homosexuales y bisexuales, se convierten en un instrumento que permite el acercamiento a estas realidades, brindando a los trabajadores sociales una fuente de información valiosa para realizar investigación y proponer proyectos de intervención que reconozcan las especificidades de estas poblaciones, permitiendo visibilizar sus necesidades y demandas.

\section{Referencias bibliográficas}

Baile, J. (2008). Estudiando la homosexualidad. Teoría e investigación. Madrid: Ediciones Pirámide.

Calderón, F. (2013). Los jóvenes y una nueva politicidad. Revista Todavía, 29. Mayo. Disponible en: http:// www.lobianco.com.ar/Clientes/todaviaweb31/29.politicanota.html

Cantú, L. Jr. (2009). The Sexuality of Migration: Border crossings and mexican inmigrant men. Nueva York: University Press.

Castello, P. y Burbano, M. (2005). Migración ecuatoriana y uso de las Nuevas Tecnologías de la Información y Comunicación. Cartillas Sobre Migración, 12. Disponible en: http://www.fes-ecuador.org/ fileadmin/user_upload/pdf/155\%20TECINFMIG122005_0437.pdf

Dirección del Sistema Nacional de Juventud "Colombia Joven”. (2013). Ley Estatutaria 1622 de 2013, Estatuto de Ciudadanía Juvenil. Recuperado de: http://www.colombiajoven.gov.co/atencionaljoven/ Documents/estatuto-ciudadania-juvenil.pdf

Dueñas, D., Pontón, P., Belzunegui, Á. y Pastor, I. (2016). Expresiones discriminatorias, jóvenes y redes sociales: la influencia del género. Revista Cientifica de Educomunicación, 46 (V). XXIV. Disponible en: https://dialnet.unirioja.es/servlet/articulo?codigo $=5297324$

Fernández, P. y Zaragoza, K. (2009). Internet y riesgo sexual en hombres que tienen sexo con hombres. Revista Gac Sanit, 23(5), 380-387. Disponible en: http://scielo.isciii.es/pdf/gs/v23n5/original3.pdf

García, C. (1989). Culturas híbridas, estrategias para entrar y salir de la modernidad. México: Editorial Grijalbo.

Guasch, O. (1993). Para una sociología de la sexualidad. Revista Española de Investigaciones Sociológicas, 64, 105-122. Disponible en: https://dialnet.unirioja.es/servlet/articulo? codigo=767350

Guasch, O. (1995). La sociedad rosa. Barcelona: Editorial Anagrama.

Hernández, C. y Alcoceba, J.A. (2015). Socialización virtual, multiculturalidad y riesgos de los adolescentes latinoamericanos en España. Revista Icono, 14(13), 116-141. Doi: 10.7195/ri14.v13i2.787.

Hidalgo, I. y Flores, A. (2008). Aquí y en el otro lado. Los significados socioculturales de la sexualidad y sus impactos en la salud sexual de los migrantes mexicanos. Revista Migraciones Internacionales, 5(3), 27-49. Disponible en: http://www.scielo.org.mx/scielo.php?script=sci_arttext\&pi$\mathrm{d}=\mathrm{S} 1665-89062008000100002$

Jacques, C., García, P., Díez, E., Martín, S. y Caylá, J. (2015). Explicaciones de las prácticas sexuales de riesgo en hombres que tienen sexo con hombres. Gaceta Sanitaria, 29(4), Disponible en: http://scielo. isciii.es/pdf/gs/v29n4/original2.pdf

List, M. (2005). Hombres: cuerpo, género y sexualidad. Revista Cuicuicol, 033, 173-202. Disponible en: http://www.redalyc.org/pdf/351/35103310.pdf

Organización Panamericana de la Salud, Organización Mundial de la Salud. (2000). Promoción de la salud sexual: Recomendaciones para la acción. (Actas de una Reunión de consulta convocada por la OPS, OMS y la Asociación Mundial de Sexología (WAS), mayo 19-22, Antigua Guatemala, Guatemala).

Restrepo, J. (2013). Sexualidades migrantes: La experiencia migratoria de los hombres homosexuales y bisexuales colombianos en España. Revista Latinoamericana de Estudios sobre Cuerpos, Emociones y Sociedades. 11. Disponible en: http://www.relaces.com.ar/index.php/relaces/article/view/204/155

Restrepo, J. (2016). Análisis comparativo de las percepciones sobre el VIH/SIDA de varones homosexuales y bisexuales colombianos, con experiencia migratoria o sin la misma. Revista de Salud Pública, 18(1). DOI: https://doi.org/10.15446/rsap.v18n1.41974

Sennet, R. y Foucault, M. (1981). Sexualidad y Soledad. Revista El Viejo Topo, 61, 47-54.

Spargo, T. (2007). Foucault y la Teoría Queer. Barcelona: Editorial Gedisa, 2ª Edición. 
Villaamil, F. y Rubio, J. (2006). Los locales de sexo anónimo como instituciones sociales: discursos y prácticas ante la prevención y el sexo más seguro entre HSH. Informe Ejecutivo. Recuperado de: http:// www.cogam.org/resourceserver/1358/d112d6ad-54ec-438b-9358-4483f9e98868/033/rglang/es-ES/filename/estudio-lsa.pdf

Weeks, J. (1998). La construcción cultural de las sexualidades. ¿Qué queremos decir cuando hablamos de cuerpo y sexualidad? En: Ivonne Szasz y Susana Lerner (comps.), Sexualidades en México. Algunas aproximaciones desde la perspectiva de las ciencias sociales (pp. 199-221). México: El Colegio de México. 